\title{
Encourager les réflexions/interactions affectives par l'usage scénarisé des outils du Web 2.0 : en quoi est-ce significatif ?
}

\author{
Sarah Anthony \\ Université McGill \\ Canada \\ Prisca Fenoglio \\ Université Paris 8 \\ France
}

\begin{abstract}
«[Because of the personal aspect] I did get more attached. [...] And so... it sort of also made it like a pet project. Like... this is ours, because it's about us»
\end{abstract}

(un étudiant de niveau A2)

\begin{abstract}
Comme bien des chercheurs contemporains l'ont signalé (Arnold 1999, 2006, 2011; Piccardo 2013; Puozzo Capron et Piccardo 2013), la filiation entre les affects et l'apprentissage des langues est indéniable. Bien que cette mise en rapport ne soit pas nouvelle, il semble qu'assez récemment l'étude des affects a repris de l'ampleur dans le domaine de la didactique des langues. La mise à l'écart de la composante affective était certainement liée à la primauté qui a été donnée à l'acquisition des savoirs, paradigme dans lequel les savoir-faire et les savoir-être passaient au deuxième plan. La tendance à valoriser l'apprentissage cognitif s'expliquerait aussi du fait qu'il parait plus facile de chercher à développer chez l'apprenant des éléments perçus comme tangibles (se prêtant donc mieux à l'évaluation) et moins intimes que ne le sont les affects. Toutefois, avec la venue et l'intérêt grandissant pour l'approche actionnelle - qui mise notamment sur les savoir-être ${ }^{1}$ - et l'apprentissage par la tâche, l'étudiant est amené à s'investir dans son apprentissage, un investissement qui par sa nature tant autoréflexive qu'interactive pourrait, à notre avis, occasionner des moments propices à explorer la composante affective.
\end{abstract}

Inspirées par ces pistes didactiques et pédagogiques, nous avons cherché à savoir si le Web $2.0^{2}$ pourrait avoir un effet sur la dyade affects/apprentissages, car certains de ces outils numériques, par leur qualité sociale, sembleraient être propices à la création d'espaces facilitants pour l'exploration des affects en salle de classe. Croyant fermement que l'utilisation de ces outils doit être scénarisée, nous nous sommes demandé si un scénario favorisant les réflexions/interactions ${ }^{3}$ affectives et faisant usage du Web 2.0 pourrait avoir des effets significatifs sur l'apprenant ${ }^{4}$. Nous avons initialement délimité deux acceptions de l'adjectif «significatif.» Dans le cadre large de notre projet ${ }^{5}$, axé sur la motivation, est «significatif» ce qui semble, aux yeux des étudiants, motivant. Les réflexions/échanges affectifs ont aussi été entendus comme «significatifs» si les apprenants et/ou les professeures y voyaient un lien avec l'apprentissage ou l'amélioration d'une compétence. Dans ces visées, un scénario intitulé «Mon Montréal à moi» a été développé.

Pendant l'année universitaire 2015-2016, ce scénario a été mis en place dans quatre sections de deux cours donnés en présentiel dans une université anglophone à Montréal, au Québec : un cours qui correspond au niveau A2 du Cadre Européen Commun de Référence pour les Langues (CECRL), un autre au niveau B2. Comme outils numériques, nous avons utilisé Etherpad, un éditeur de texte collaboratif qui offre un espace pour rédiger des documents en ligne de manière synchrone et/ou asynchrone, et un autre pour clavarder. Nous avons également fait usage de Google+, un réseau social et une plateforme de partage qui ressemble beaucoup à Facebook quant à son fonctionnement ${ }^{6}$.

La séquence pédagogique comptait plusieurs étapes. Tout d'abord, en utilisant Etherpad, les étudiants ont rédigé de façon coopérative et/ou collaborative un scénario de film portant sur leur lien affectif quant à un endroit montréalais choisi. Pendant la rédaction de ce texte, ils ont clavardé pour faire leurs remue-méninges et pour discuter des corrections à apporter au scénario. Après avoir tourné et monté leur film, ils l'ont affiché sur la page Google+ de la classe, ont fait des commentaires en français autour des vidéos, pour ensuite voter pour les trois meilleures (fonction 1+). En faisant ce projet, les étudiants devaient aussi répondre à certaines exigences :

1. Ils étaient amenés à choisir un objet qui représente la vie à Montréal pour tous les membres du groupe et celui-ci devait être présent dans la majorité des séquences vidéo

2. Ils avaient à faire une présentation personnelle de leurs impressions et de leurs sentiments d'attachement au milieu dans lequel ils vivent. Pour cette raison, ils étaient encouragés à incorporer au scénario une phrase de type : "Mon Montréal à moi c'est... parce que je + émotion. » 
Anthony, Sarah et Prisca Fenoglio. « Encourager les réflexions/interactions affectives par l'usage scénarisé des outils du Web 2.0 : en quoi est-ce significatif ? » Nouvelle Revue Synergies Canada, No¹1 (2018)

La première contrainte visait à assurer une meilleure cohésion et à encourager un esprit de partage au sein de chaque groupe, tandis que la deuxième avait pour objectif de donner à l'affectif un espace légitime dans la classe de FLS. Soulignons également que la mise en place de contraintes est souvent propice au développement de la créativité (Muller 92), une des visées de notre séquence pédagogique. Hormis ceci, le scénario a été conçu de sorte à donner une grande liberté à l'apprenant quant aux choix à faire pour en arriver au produit final, le film.

Les objectifs d'apprentissage de notre scénario étaient multiples et visaient à améliorer, par ordre d'importance :

1. Les savoir-être : il s'agissait de conduire l'étudiant à être ouvert à ses affects vis-à-vis du lieu montréalais choisi et à la possibilité de les exprimer, tout en étant réceptif à l'autre et au partage.

2. Les savoir-faire : l'objectif était d'amener l'apprenant à savoir mieux créer, collaborer, apprendre à l'extérieur de la classe, à se corriger (ainsi qu'autrui) à l'écrit et à développer sa littératie numérique. 3. Les savoirs : il était important de chercher à améliorer les compétences orales et écrites (français standard et familier).

Cet éventail de visées pédagogiques montre que notre vision de l'enseignement est holistique, ne perdant pas de vue le rôle central des facultés cognitives de l'apprenant, mais tenant compte des aspects physiques et affectifs en même temps.

\section{Éléments théoriques et méthodologiques}

\section{Affects et apprentissage/enseignement des langues}

Ce travail prend sa source d'inspiration dans les recherches d'Arnold, dont la vision holistique de l'enseignement fait écho à la nôtre. De ses écrits, nous retenons plusieurs notions, notamment la définition qu'elle donne aux affects, soit : «un large domaine qui comprend les sentiments, les émotions, les croyances, les attitudes et qui conditionne de manière significative notre comportement» («Comment les facteurs» 407). Elle souligne que les aspects affectif et effectif de l'apprentissage sont liés et évoque le lien établi par Rodríguez et al., ou encore Dörnyei entre affects positifs et motivation.

En référence au développement de la compétence communicative, Arnold fait appel au modèle de Mclntyre et al., au sein duquel l'utilisation de la langue seconde à des fins communicatives se situe au sommet de la pyramide et dépend de la disposition à communiquer de l'apprenant. Elle note que les quatre niveaux inférieurs de cette pyramide ont un rapport avec l'affectif, montrant ainsi l'importance de cette composante pour que l'apprenant veuille s'exprimer dans la langue seconde. Comme Arnold, nous tenons compte de la primauté de la variable affective pour l'amélioration de la compétence communicative et, de façon plus large, pour le succès dans l'apprentissage des langues. Les implications qu'elle mentionne sur le travail en salle de classe (rencontre des autres, coopération, variété et vraisemblance des activités, etc.) correspondent à notre vision de praticiennes. Ainsi, «Mon Montréal à moi» amène l'apprenant à exprimer le lien affectif qu'il porte à son milieu de vie, ceci par le biais d'un travail coopératif et/ou collaboratif, créatif et ayant lieu partiellement en dehors du cadre de la classe. Ce scénario vise à créer un climat socio-affectif propice à la confiance, à l'expression et à l'apprentissage de la langue, dans la lignée des travaux d'Arnold.

\section{Affects, médiation technologique/Web 2.0 et scénarisation}

Les travaux d'Arnold mentionnés ne prennent pas en compte un élément présent dans notre scénario : la médiation technologique. De nombreuses recherches (par ex. celle de Jézégou) se penchent sur la dimension sociale, ou socio-affective, au sein de la médiation technologique, mais dans des dispositifs d'enseignement à distance. Certaines (comme celle de Rémon) font écho aux travaux d'Arnold quant à la création d'un climat social propice à l'apprentissage. Par contraste, nous sommes dans le contexte d'un apprentissage collectif assisté par ordinateur (ACAO), par l'entremise des outils du Web 2.0, qui a lieu majoritairement en présentiel. Dans le champ de l'apprentissage/enseignement des langues, ces outils ont été étudiés, notamment dans leurs liens avec l'approche actionnelle (voir Dejean-Thircuir et Nissen) ou par le biais de la collaboration et de l'ouverture possible vers d'autres communautés afin de faire de l'apprenant un acteur social (voir Ollivier et Puren). Nous nous intéressons ici au fait que ces outils permettent le développement de la créativité (par l'écriture, la vidéo, le montage) et des interactions sociales (la coopération et/ou la collaboration [Etherpad], ainsi que le partage et les commentaires sur les productions [Google+]). 
Anthony, Sarah et Prisca Fenoglio. « Encourager les réflexions/interactions affectives par l'usage scénarisé des outils du Web 2.0 : en quoi est-ce significatif ? " Nouvelle Revue Synergies Canada, No¹1 (2018)

Signalons que Kreijns et al. identifient deux pièges potentiels aux interactions sociales en ACAO. Le premier est de tenir les interactions sociales pour acquises. Cependant, même avec des outils les favorisant (comme le Web social), elles ne sont pas une évidence. Pour cette raison, la scénarisation pédagogique nous semble essentielle pour encourager des interactions stratégiques entre les apprenants. Nous suivons en cela la définition de Dejean-Thircuir et al., qui place les interactions au cœur de la scénarisation pédagogique : «une ou plusieurs tâche(s) combinée(s) avec un scénario de communication et prévoyant une chronologie des échanges» (Mangenot 19 [italiques de l'auteur]). Le deuxième piège est de limiter les interactions sociales à l'accomplissement de tâches et au processus cognitif. Or les échanges éducatifs sont normalement plus fructueux si un lien de confiance a été établi auparavant entre les apprenants. Le développement de cette confiance, grâce aux interactions sociales, peut donc prendre place en parallèle au processus cognitif, et ainsi le soutenir, ce que nous recherchons.

\section{Quelques éléments méthodologiques}

Notre position est celle d'observatrices participantes, puisqu'à la fois professeures et actrices de cette recherche. II s'agit d'adopter, dans cette analyse d'une situation intégrant des acteurs humains et non-humains (les outils), une démarche compréhensive, avec des explorations empirique et théorique conduites dans une logique dialectique, au sein d'un paradigme qualitatif.

L'objectif de notre premier recueil de données, effectué au printemps 2016, était large. Il s'agissait d'une part de comprendre l'expérience vécue par les apprenants par le biais de notre scénario et à la lumière des objectifs de notre projet (voir la note 5). D'autre part, nous souhaitions analyser quel type de discours se développait à travers les traces des interactions écrites (commentaires) sur Google+, afin de comprendre dans quelle mesure les interactions affectives étaient encouragées (par la tâche et par l'outil) et quels effets cela pouvait avoir sur les dynamiques de groupes et sur l'apprentissage.

Comme le détaille le tableau suivant, nous avons recueilli et analysé trois types de données provenant des apprenants : des questionnaires anonymes ${ }^{7}$, des entretiens ${ }^{8}$ et leurs commentaires sur Google+.

\begin{tabular}{|c|c|c|c|}
\hline Participants & Questionnaires & Entretiens & Traces sur Google+ \\
\hline \multirow{3}{*}{$\begin{array}{l}\text { - âgés de } 18 \text { à } 22 \\
\text { ans (majoritairement } \\
\text { entre la } 1^{\text {It }} \text { et la } 4 \\
\text { année d'université) } \\
\text { - provenant de } 4 \\
\text { classes différentes, } \\
\text { soient } 90 \text { étudiants } \\
\text { au total ( } 63 \% \text { de } \\
\text { femmes et } 37 \% \\
\text { d'hommes) }\end{array}$} & A2 : 41 étudiants & A2: 5 étudiantes & $\begin{array}{l}\text { A2: } 114 \text { commentaires } \\
\text { analysés }\end{array}$ \\
\hline & B2 : 46 étudiants & $\begin{array}{l}\text { B2: } 4 \text { étudiants, dont } 2 \\
\text { hommes et } 2 \text { femmes }\end{array}$ & $\begin{array}{l}\text { B2:184 commentaires } \\
\text { analysés }\end{array}$ \\
\hline & $\begin{array}{l}\text { TOTAL : } 87 \\
\text { questionnaires }\end{array}$ & $\begin{array}{l}\text { TOTAL : } 9 \text { personnes } \\
\text { interrogées au cours de } \\
4 \text { entretiens de } 2 \text { ou } 3 \\
\text { étudiants }\end{array}$ & $\begin{array}{l}\text { TOTAL : } 298 \\
\text { commentaires analysés }\end{array}$ \\
\hline
\end{tabular}

Parmi les 87 enquêtés par questionnaire, nous avons sollicité 9 personnes volontaires pour participer à des entretiens semi-directifs en groupes de 2 ou 3 d'une trentaine de minutes, qui visaient avant tout à approfondir les thèmes abordés dans le questionnaire. Nous avons complété ces «pratiques déclarées» (Clanet 328) par des «productions invoquées» (Van der Maren, Méthodes 82) - l'analyse des traces des interactions sur Google+ -, afin de voir le lien entre ce qui est dit et ce qui est fait. Pour les entretiens et les traces, nous avons procédé par analyses thématiques, faites à partir de l'attention portée aux thèmes émergents et au matériau linguistique (occurrences lexicales, positionnements énonciatifs, modalisation, etc.). L'outil méthodologique utilisé est l'analyse de discours, avec procédure ouverte. Nos propres observations de praticiennes ont également été prises en compte. Elles ont une valeur empirique et concernent avant tout les réactions des étudiants lors du partage du produit final et nos évaluations quant aux produits finals eux-mêmes.

Cette démarche de recherche a un caractère exploratoire. Elle a été construite dans l'idée d'aboutir à une « triangulation élargie, " visant « non pas à confronter des données, mais à enrichir des données obtenues par un instrument favorisant certaines expressions par celles obtenues par un autre instrument stimulant d'autres facettes du discours ॥ (Van Der Maren, «Les recherches » 76). L'objectif est de s'approcher avec le plus de richesse et de nuance possible d'une réponse à la problématique de départ, à savoir si un scénario 
Anthony, Sarah et Prisca Fenoglio. « Encourager les réflexions/interactions affectives par l'usage scénarisé des outils du Web 2.0 : en quoi est-ce significatif ? " Nouvelle Revue Synergies Canada, No¹1 (2018)

encourageant l'expression affective et faisant usage du Web 2.0 peut être significatif en termes d'expérience vécue par les apprenants et/ou les professeures quant à la motivation et/ou l'apprentissage.

\section{La dyade affectif/significatif : les résultats ${ }^{9}$}

\section{La composante affective : un élément très apprécié du scénario}

Nous avons posé la question suivante pour chaque composante du scénario (la collaboration, la créativité, les affects et le hors-cadre) : "How did you feel about the part of the activity that was collaborative, creative, etc.? " Les apprenants avaient le choix entre "It was motivating» ou compléter " It was.... » Ensuite, on leur demandait d'expliquer leur réponse. Nous avons remarqué que la composante affective n'était pas perçue comme un facteur clé de motivation. Aux niveaux A2 et B2, les résultats quantitatifs des questionnaires révèlent que l'élément affectif s'est classé au $3^{\mathrm{e}}$ rang quant au rapport entre chacune de nos variables et la motivation.

\begin{tabular}{|l|l|l|l|l|}
\hline Groupes & Collaboration & Créativité & $\begin{array}{l}\text { Aspect personnel } \\
\text { et partage des } \\
\text { sentiments }\end{array}$ & Hors-cadre \\
\hline A2 & $56 \%(\# 2)$ & $53 \%(\# 4)$ & $55 \%(\# 3)$ & $60 \%(\# 1)$ \\
\hline B2 & $78 \%(\# 1)$ & $76 \%(\# 2)$ & $74 \%(\# 3)$ & $54 \%(\# 4)$ \\
\hline
\end{tabular}

Par contre, les données qualitatives - provenant des questionnaires et des entretiens - soulignent que cette composante engendre le moins de commentaires négatifs ${ }^{10}$; c'est aussi la seule qui n'a pas paru "difficult / difficile.» Qui plus est, par rapport aux autres variables observées (l'usage des outils, la collaboration, la créativité, le hors-cadre), il y a très peu de traces de dualité dans les réponses (ex. "but / mais,» "however / cependant,» etc.). Ceci indique que les apprenants avaient une impression positive et non ambivalente de cette variable. Ainsi, même si elle n'est pas identifiée comme étant la plus motivante, la composante affective est appréciée par les apprenants.

\section{Le développement de savoir-être : l'ouverture à la connexion et à la découverte}

L'idée que le scénario, doté de sa composante affective, ait amené les étudiants à développer des savoir-être, notamment en promouvant une ouverture à la connexion et à la découverte à l'égard de soi, des autres, de la ville, de la culture et de la langue, est revenue à maintes reprises dans les données suscitées. La reprise parfois anaphorique de phrases comme «lt + verb + me» / «ll + me + verbe,» dans lesquelles le scénario ou bien l'aspect affectif de celui-ci (souvent représenté par «it» ou «il») est doté d'une agentivité, souligne l'importance de la scénarisation pour l'approfondissement des savoir-être en question. Dans les réponses au questionnaire ${ }^{11}$, ceci se manifeste au niveau A2 par des phrases comme : «lt motivated me to continue French to better experience the places I love in Montreal. It helped me connect with classmates.» Le même phénomène se présente au niveau B2 : «Il m'a fait penser et réfléchir, donc ça c'est bon» // "L'activité me connecte avec la ville.» Cet aspect est moins présent dans les entretiens, cependant en voici un exemple :

[RL/B2] : I really liked the personal aspect 'cause it... it sorta make - make - how would you say that. [...] it gets you to reflect on, like, your relationship with the city. Which is not something you would do, like, every day. And I thought that was really, it was pretty powerful for me 'cause I talked about language, the language here in Montreal. The French language. [...] But yeah, in general I thought it was a good activity to get us to, like, think about what the city means to us.

Hormis l'agentivité qui est donnée à deux reprises au scénario ou à l'aspect affectif de celui-ci dans l'aide à la découverte de la ville, notons l'emploi de l'adjectif « powerful, » décrivant l'effet de l'activité affective, ici lié au langage, sur le participant. Vu la récurrence de ce type de propos, nous proposons une troisième acception au terme "significatif, " qui est non seulement en rapport avec la motivation et les apprentissages, mais aussi rattachée à des expériences décrites comme " marquantes » par les apprenants, et qualifiées par des adjectifs à valeur emphatique, comme cet exemple du niveau A2 en témoigne : " sharing my feelings about Montreal was a really good thing for me... almost therapeutic. » 
Anthony, Sarah et Prisca Fenoglio. « Encourager les réflexions/interactions affectives par l'usage scénarisé des outils du Web 2.0 : en quoi est-ce significatif ? " Nouvelle Revue Synergies Canada, No¹1 (2018)

Si les données suscitées signalent l'importance de la scénarisation et l'appréciation des apprenants de celle-ci pour approfondir ces savoir-être que sont la capacité à la connexion et à la découverte, nos observations de praticiennes montrent que cet approfondissement a eu lieu. Ainsi, nous avons constaté, entre autres, l'investissement des apprenants dans cet exercice, par le biais de la complexité de certaines productions $^{12}$. Cela reflète-t-il la profondeur du rapport personnel qu'ont les apprenants avec Montréal ? II semble légitime de soulever la question. Le fait que cet intérêt pour la découverte est non seulement orienté vers soi, mais aussi vers les autres et vers Montréal a, quant à lui, paru au travers de leurs interactions avec le milieu francophone dans le produit final et de leur enthousiasme pour le partage des vidéos.

\section{Le développement d'un savoir-faire : apprendre à parler de soi}

«Mon Montréal à moi,» en offrant un temps et un encadrement propices à l'exploration des savoir-être susmentionnés, est perçu par les étudiants et les professeures comme soutenant, de ce fait, l'approfondissement d'un savoir-dire à partir d'un sujet personnel. Ceci est souligné à plusieurs reprises par les participants au niveau A2 : «lt felt more useful learning words to talk about my own experience» et dans les entretiens, ils notent : [GS-G] «you might as well learn the vocabulary [to talk about things that are personal] that you would wanna use.» Cette prise de conscience du développement du savoir-dire au niveau A2 est certainement liée au fait que les capacités lexicales et d'expression orale sont plus limitées que celles des étudiants de niveau B2. Toutefois, si l'amélioration d'un savoir-dire à partir d'un sujet personnel est remarquable pour les étudiants du niveau A2, l'aisance linguistique favorisée par le fait de parler de ce qu'on aime ou de ce qui est personnel est ressentie par tous. Au niveau B2, les apprenants font les constats suivants en lien avec l'aspect personnel du scénario : «il me force de ne pas être gêné» // "c'était facile de m'exprimer.» Pareillement, au niveau A2, les participants signalent que "this made speaking much easier» // "[it is] beneficial in helping us express ourselves in another language.»La même tendance est présente dans cet extrait d'entretien : "[MP/B2] I feel like you're usually more comfortable speaking about stuff that you like. And if you want to say something good about it, I think those kind of words come to us quicker than thinking about something to do on a test, for example.» La pluralité des exemples accentue l'importance du lien à faire entre parler de ce qu'on aime et l'aisance linguistique identifiée par les apprenants, évoquée ci-dessus, par une certaine impression d'aisance («more comfortable»), de facilité («easier») et de fluidité linguistique («words come to us quicker»). Toutefois, même si parler de ce qu'on aime semble faciliter cette aisance, ils reconnaissent l'importance du cadre pédagogique («il [l'aspect personnel] me force») qui les guide vers des sujets plus personnels, voire affectifs, qui ne sont pas typiquement abordés en salle de classe. Comme professeures, nous avons remarqué une assez bonne amélioration de la fluidité linguistique se manifestant dans les choix des étudiants quant au tournage de la vidéo. Au niveau B2, la grande majorité des étudiants ont choisi de parler devant la caméra de préférence à la voix off et le débit des apprenants est généralement naturel, il y a peu de mémorisation et de lecture de textes. Au niveau A2, la plupart des étudiants ont décidé de s'exprimer face à la caméra de manière fluide et vivante, mais on note parfois de la lecture ou de la mémorisation $^{13}$.

Ainsi, il semblerait y avoir, chez les étudiants, une prise de conscience de l'importance du scénario pour l'approfondissement des savoir-être et savoir-faire visés, et, dans nos observations de praticiennes, des preuves que les apprentissages désirés ont eu lieu.

\section{La triade affectif/Web 2.0/significatif : les résultats ${ }^{14}$}

Nous nous sommes penchées sur l'usage de Google+, qui nous a paru encourager la dimension affective du scénario ${ }^{15}$, afin de voir si l'emploi de cet outil était significatif. Rappelons que Google+ a été utilisé pour partager, commenter et voter pour les productions finales.

\section{Le partage et le vote sur Google+ : un incitatif positif}

Selon les questionnaires ${ }^{16}$ et les entretiens, le fait de partager leurs productions, voire d'être en compétition, par le biais de Google+, a eu plusieurs conséquences liées aux affects. Cela a tout d'abord été apprécié, particulièrement au niveau B2. Sont mentionnés à la fois les votes, les commentaires et les interactions, ceci dans les questionnaires «j'aimais avoir la possibilité de voter et commenter les vidéos de mes amies» (B2), tout comme dans les entretiens : [SCS/A2] «I also liked that we could, we had to make comments on the videos.» Ce dernier commentaire est intéressant en ce qu'il y a hésitation entre la possibilité («we could»), corrigée par la contrainte («we had to»), et que cette dernière est mise en lien avec l'appréciation. 
Anthony, Sarah et Prisca Fenoglio. « Encourager les réflexions/interactions affectives par l'usage scénarisé des outils du Web 2.0 : en quoi est-ce significatif ? " Nouvelle Revue Synergies Canada, No¹1 (2018)

Toujours au niveau B2, cette appréciation revêt aussi la forme d'une émulation positive, face au [DV/B2] «competitive edge» créé : [RL/B2] «the voting system and so... I thought that was REALLY smart 'cause it gave us, like - that's like our main source of motivation, I would say, like, 'cause... we wanted to get the final votes. And so, 'cause if every, like, if there wasn't really any competition, we would just be like "Yeah, let's make a video, [it] doesn't have to be great".» Cet exemple fait un lien explicite entre la qualité recherchée de la vidéo, la compétitivité et le désir de bien faire. Cette émulation positive n'est pas sans rapport avec une certaine pression liée au partage, qui a encouragé les apprenants à faire de leur mieux pour oser montrer leurs productions : [DV/B2] «there was definitely some pressure, I guess, ah, because we had the idea that we would be sharing the video also to people in the classroom when we reconvened.» On remarque la modalisation emphatique de ce commentaire (adverbe en gras), toutefois tempérée par l'expression «I guess.» Ainsi, Google+ constitue un incitatif positif pour les étudiants, particulièrement au niveau B2 : il est apprécié pour son côté compétitif (les votes) et pour le fait de «rendre publiques,» par le partage, les productions finales.

\section{Le développement de savoir-être ouvert à l'autre et inclusif}

Dans le prolongement des réflexions concernant l'ouverture à la connexion et à la découverte de l'autre, on peut dire que Google+, une plateforme qui promeut le partage, a créé un terrain fertile pour la réceptivité quant à autrui, voire le développement d'un savoir-être inclusif. Au niveau A2, le partage a fait levier à l'ouverture : "seeing other students openness encouraged me to be more open" // "good to share my nice memories with my peers.» On note ici l'appréciation (good) du partage, l'aspect personnel de l'activité (le possessif "my» utilisé deux fois), qui, peut-on supposer, rend les pairs plus proches (my peers). Au niveau B2 est mentionnée par plusieurs la création de liens entre les étudiants (par de nouveaux sujets de conversation : les vidéos) : [RL/B2] «I would be conversing with people across the room about their videos. [...] so it kind of helped establish solidarity, I guess.»11

II est intéressant de noter qu'on retrouve cette appréciation (du travail de l'autre), ce désir d'être inclusif et cette recherche de rapprochement dans les traces d'interactions sur Google+. La consigne orientait vers l'affectif et le positif, dans la mesure où nous demandions aux apprenants de commenter les vidéos qu'ils avaient le plus aimées. Toutefois, l'outil, accompagné de sa culture d'usage (Thorne $[2003]^{18}$ ), a aiguillé vers le positif (+1) et l'affectif. Les messages sont fortement orientés vers l'inclusion par l'expression de l'appréciation, l'empathie, l'adhésion (on note ainsi les apostrophes, les émoticônes et la récurrence de l'adverbe "aussi»: j'aime/j'adore aussi, pour moi aussi), l'annonce d'action subséquente au visionnage de la vidéo (ex. maintenant je veux aller / je dois l'essayer), voire la narration d'un souvenir en lien avec ce qui est partagé. L'expression laudative et emphatique positive devient une nouvelle norme, comme le montrent le nombre de points d'exclamation, d'interjections ou encore les phrases très modalisées par des adverbes, des adjectifs (ex. adorable, parfait, excellent, etc.) et des verbes emphatiques (ex. j'étais impressionné). II y a également une recherche de proximité affective et dialogique: phrases affectueuses, prénoms dans la réaction, émoticônes sont là pour en témoigner. Cependant, le plus souvent, ce dialogue n'aboutit pas (les questions restent sans réponses). En ce sens, l'observation des commentaires sur Google+ donne l'impression d'une salle pleine de vie, mais où les gens parleraient tous en même temps, ce qui n'empêche pas les liens sociaux de se créer...

Ainsi, l'affectivité des apprenants envers ces partages et ces votes est nette et peut avoir une coloration différente selon les niveaux. Ceci émerge des questionnaires, des entretiens et des commentaires, mais les traces interactionnelles sur Google+ la mettent en pratique. En ce qui concerne l'aspect significatif, on semble osciller entre la motivation (le désir de bien faire pouvant en constituer un levier), l'apprentissage (par l'ouverture à l'autre et/ou, son corolaire, la création de liens) et l'expérience de partage vécue (comme en témoigne son appréciation), les trois pôles du terme «significatif» émergeants de cette étude.

\section{Le questionnement et le développement de savoir-dire de façon informelle}

Dans les questionnaires et entretiens, les étudiants ont évoqué les commentaires de Google+ sous l'angle de l'apprentissage de la langue, dans un rapport affectif et ambivalent à la norme et à l'usage familier du langage. Certains font part de leur peur de l'erreur, de manière, encore une fois, fortement modalisée, ce qui reflète une crainte, comme on le voit ici : [SP/A2] : «Yeah, I'm always scared to make mistakes» ou [MP/B2] : «And I feel like in French, I feel a little bit more uncomfortable BECAUSE I don't know the colloquial terms. So I feel like I either sound really lame because everything's really perfect but I don't know how else to write.» D'autres, au contraire, se sentent plus libres, moins sous pression par rapport à la norme : [DV/B2] : "chatting online [...] I think that it was easier to -- there was less pressure [...] I didn't feel the pressure of making a mistake [...], 
Anthony, Sarah et Prisca Fenoglio. «Encourager les réflexions/interactions affectives par l'usage scénarisé des outils du Web 2.0 : en quoi est-ce significatif? " Nouvelle Revue Synergies Canada, No¹1 (2018)

because I would do that in English anyways.» Ce sentiment de liberté se reflète parfois, aux deux niveaux, mais surtout au niveau $B 2^{19}$, par une plus grande prise de liberté linguistique, comme l'illustre le registre de langue utilisé dans le commentaire suivant : G+/A2 «ouai chuis d'accord avec izabella. jpense que le style de video est tres charmant. et vous avez parlé aux yeux des tous!!! j le peux pas faire haha.» Cet exemple montre le lien entre la culture d'usage de l'outil utilisé qui invite à la langue familière et les tentatives d'appropriation de celle-ci par les étudiants. L'outil entraine un questionnement et la prise de risque nécessaire à l'apprentissage d'un savoir-dire nouveau, familier, en L2.

\section{Conclusion}

Arrivées au terme de cette étude, que pouvons-nous dire des effets de l'encouragement - par la scénarisation pédagogique et les outils du Web 2.0 - des réflexions/interactions affectives sur nos étudiants de français langue seconde? En quoi sont-ils significatifs?

(1) En ce qui concerne la motivation, la dimension affective n'était pas la plus significative, mais a été appréciée.

(2) En termes d'apprentissages, par le biais de la composante affective, se sont développés des savoir-être qui encouragent l'ouverture à la connexion et à la découverte à l'égard de soi, des autres, de la ville, de la culture et de la langue. Cela a entrainé le développement d'un savoir-dire à partir d'un sujet personnel. Grâce aux partages que Google+ a suscités, les apprenants ont aussi travaillé sur leur capacité à être ouvert et inclusif, par la création d'une communauté hors ligne (par de nouveaux sujets de conversation : les vidéos) et en ligne. Cette communauté en ligne s'avère nettement orientée vers l'affectif car Google+, avec ses particularités fonctionnelles et son code d'usage, prolonge et amplifie l'intention socio-affective du scénario. Enfin, un questionnement sur le savoir-dire de manière informelle dans les commentaires sur Google+ et sur l'aisance par rapport à ce type de discours a émergé ainsi que des tentatives d'appropriation de ce langage familier propre aux commentaires.

(3) En ce qui a trait à l'expérience vécue, l'activité a, à plusieurs reprises, été identifiée comme une expérience marquante. L'encouragement des réflexions/interactions affectives au moyen de la scénarisation mène aussi à un attachement au projet, à une fierté vis-à-vis du produit final et rend le projet significatif, car personnel, lui donnant du sens.

L'importance de la scénarisation quant à l'exploration de la composante affective gagne à être soulignée et remise en contexte. En effet, comme le développe Mangenot dans le cadre de l'apprentissage/enseignement à distance, pour bien cerner l'effet du scénario sur l'apprenant, il faut considérer celui-ci d'un point de vue systémique et tenir compte de plusieurs paramètres : les caractéristiques sémio-pragmatiques des outils, l'enjeu de la communication, la relation entre apprenants et tuteurs et la valeur cognitive, culturelle, affective des contenus échangés. Le scénario est donc une composante parmi plusieurs qui puissent participer au processus complexe qu'est l'apprentissage d'une langue seconde et le rendre significatif.

Quoique la mise en place du scénario «Mon Montréal à moi» ait mené généralement aux résultats escomptés, cette recherche a certaines limites. En raison de sa nature exploratoire et de son appartenance à un projet de recherche plus global sur la motivation, la problématique choisie et la récolte de données qui en a découlé se sont avérées très larges et profiteraient d'un certain resserrement lors d'une deuxième itération éventuelle du scénario, ainsi que dans le développement de prochains scénarios. De nature principalement qualitative, cette étude pourrait être enrichie par une quantification de l'aspect significatif de la scénarisation sur les apprentissages, notamment par l'ajout de pré-tests et de post-tests. À l'avenir, nous comptons élargir la passation des entretiens à des apprenants dont l'engagement dans le projet semblerait, à premier abord, moindre, afin de réfléchir aux améliorations à apporter au scénario. Ces entretiens nous permettront de mieux pallier aux limites du scénario et de sa mise en pratique relevées dans nos questionnaires (voir la note 10).

Finalement, «Mon Montréal à moi» met les savoir-être de l'avant, est soutenu par des outils qui prolongent notre vision holistique de l'apprentissage/enseignement et éveille des questionnements quant aux rapports, notamment, aux cultures d'usage et à la norme linguistique. Par le développement de savoir-être ouvert et inclusif, il nous semble que le rapport à soi et à l'autre est travaillé, voire modifié, créant un terrain propice pour faire levier aux questionnements et aux apprentissages de savoir-faire et de savoirs. À la lumière de ces résultats encourageants pour l'apprentissage d'une langue seconde, explorer les possibilités offertes par le Web 2.0 pour intégrer la variable affective dans la scénarisation pédagogique nous semble une piste intéressante pour les praticiens. 
Anthony, Sarah et Prisca Fenoglio. « Encourager les réflexions/interactions affectives par l'usage scénarisé des outils du Web 2.0 : en quoi est-ce significatif ? " Nouvelle Revue Synergies Canada, No¹1 (2018)

\section{Contributions}

Sarah Anthony et Prisca Fenoglio ont contribué également à cette étude.

\section{Notes}

1 Le Cadre Européen Commun de Référence pour les Langues (CECRL) fait ressortir l'importance de la composante affective dans l'apprentissage des langues en indiquant, dans la section portant sur le savoir-être, que : "L'activité de communication des utilisateurs/apprenants est non seulement affectée par leurs connaissances, leur compréhension et leurs aptitudes mais aussi par des facteurs personnels liés à leur personnalité propre et caractérisés par les attitudes, les motivations, les valeurs, les croyances, les styles cognitifs et les types de personnalité qui constituent leur identité » (84).

2 Selon Zourou (2012), le Web 2.0 est une plateforme technologique. Celle-ci permet aux applications du Web social « de se mettre en œuvre grâce aux possibilités qu'ont les utilisateurs de créer, distribuer, partager et manipuler différents types de contenus, la plupart accessibles à tous librement » (parag. 5). Le Web social fait donc partie du Web 2.0. Nous avons utilisé, dans cet article, l'expression Web 2.0 pour référer aux différentes applications du Web social que nous avons utilisées.

3 Par «réflexions affectives,» nous entendons des réflexions quant à ses propres émotions et nous définissons le terme «interaction affective» comme une interaction sociale qui fait usage du registre émotionnel (par exemple, discuter de comment on se sent face à l'apprentissage d'une langue seconde).

4 Nous avons été inspirées par la formule suivante, proposée par Kreijns et al. (2003) décrivant une expérience d'apprentissage désirable : "Valued Learning Experience = F [Pedagogy, Content, Community]» (342). Ils soulignent que chacune des trois variables de cette formule doit être prévalente (selon les auteurs, une pédagogie fonctionnelle, des contenus pertinents et une communauté soudée) pour créer cette «Valued Learning Experience,» un apprentissage recherché par l'apprenant et l'enseignant. Cet objectif est proche du nôtre (nous cherchons ce qui peut rendre l'apprentissage «significatif»). Ainsi, nous pourrions proposer une formule proche de la leur : Significatif = F [Scénario, Affects, Outils], sachant que "Scénario» comprend leurs notions de «Pedagogy» et "Content» et que d'actionner les «Affects» renvoie à la création de la communauté, toujours selon ces mêmes auteurs, et tout ceci accompagné de la médiatisation par les «outils» du Web 2.0.

5 Le scénario qui fait l'objet de la présente étude constitue un des 11 scénarios/activités pédagogiques que nous avons développés depuis 2015 au sein de notre projet de recherche intitulé «Inno-moti-vation» (innomotivation.wordpress.com/), qui s'articule autour de la question suivante : qu'est-ce qui motive les apprenants à approfondir leurs connaissances, et idéalement à s'engager dans leurs apprentissages ? À cette question très large, nous proposons que l'utilisation scénarisée du Web 2.0 peut encourager la motivation (et donc approfondir l'apprentissage) en cultivant la créativité, les expériences hors-cadre, le développement d'émotions favorisant l'apprentissage ainsi que les compétences de collaboration. Qui plus est, ce type de scénario est propice au développement de la littératie numérique.

6 Etherpad ( public.etherpad-mozilla.org/) permet à chaque participant d'identifier son travail par une couleur et un nom ou un pseudonyme. Cet outil a aussi été choisi pour sa facilité d'usage (il ne requiert ni installation ni inscription) et afin d'inciter les apprenants à développer leur littératie numérique, en les invitant à découvrir un outil qu'ils ne connaissaient que peu ou pas. Le choix de Google+, qui requiert une inscription, visait le partage, les commentaires et le vote (fonction +1 ) sur les productions finales et à amener les apprenants à développer leur littératie numérique. Nous cherchions également à ne pas empiéter sur la vie sociale personnelle de l'étudiant en évitant d'utiliser un outil comme Facebook qui aurait rendu la distinction entre les sphères intime et éducative plus confuse. Signalons que presque $100 \%$ des étudiants participants disent utiliser les outils du Web 2.0 de façon fréquente. Ce sont des utilisateurs réguliers d'une variété d'outils dans plusieurs contextes qui ont déjà une assez bonne littératie numérique. Toutefois, très peu d'entre eux connaissaient Etherpad et Google+.

7 Le questionnaire, approuvé par le bureau d'éthique de McGill (voir REB III : www.mcgill.ca/research/researchers/compliance/human/reb-i-ii-iii), comportait huit parties. Cinq d'entre elles visaient principalement à questionner le lien entre différents éléments du scénario (les outils, la collaboration, la créativité, les affects, l'apprentissage hors-cadre) et la motivation des apprenants. Les trois autres étaient plus générales et concernaient leurs apprentissages, leur appréciation et leur motivation globale pour le projet. Le questionnaire complet est en annexe.

${ }^{8}$ Notre guide d'entretien, également approuvé par le bureau d'éthique de McGill, est composé de questions ouvertes plutôt générales, centrées sur les pratiques et représentations des sujets interrogés et aborde les mêmes thèmes que le questionnaire, ceci afin de les développer et les préciser. 
Anthony, Sarah et Prisca Fenoglio. « Encourager les réflexions/interactions affectives par l'usage scénarisé des outils du Web 2.0 : en quoi est-ce significatif ? » Nouvelle Revue Synergies Canada, No¹1 (2018)

${ }^{9}$ Afin de ne pas alourdir le texte, nous avons fait les choix rédactionnels suivants : quand la citation provient des entretiens, nous avons mis les initiales de la personne interrogée et son niveau ex. [KW/A2] avant la citation. Quand la citation vient des questionnaires, les deux barres obliques (//) signalent le changement de répondant et nous avons mis le niveau entre parenthèses si nécessaire : ex. (B2), après la citation. Quand la citation vient des traces sur Google+, nous avons mis G+/niveau devant celle-ci.

10 II y a trois commentaires négatifs qui sont survenus aux deux niveaux dans les questionnaires et les entretiens : l'aspect chronophage de l'activité, le problème de pondération de celui-ci (pas assez de poids donné à cette évaluation) et son timing (certains auraient préféré que l'activité se fasse au premier trimestre).

11 Pour cette partie de nos résultats, qui concerne le lien entre la réflexion/interaction affective et la dimension significative, nous nous sommes penchées particulièrement sur la quatrième question du questionnaire, qui traite de l'aspect affectif : How did you feel about the part of the activity that was affective? (i.e. the personal aspect of the activity and the sharing of your feelings for a place that is special to you in Montreal)? You can choose 2 answers. It was motivating / It was... Please explain your answer. Le questionnaire complet est en annexe.

12 Nous pensons notamment à une vidéo au niveau B2 de style «animation technique» et à d'autres aux deux niveaux qui présentaient des montages musicaux et narratologiques (tissage de dialogues, monologues, voix off, etc.) complexes.

13 La mémorisation a été mentionnée en entretien par certains étudiants et avait pour but de parler de manière fluide face à la caméra, d'enrayer leur stress et de faire moins d'erreurs. Ceci n'était pas l'objectif de l'activité mais n'a pas donné lieu à des productions orales paraissant inauthentiques et récitées dans les produits finals. Cela a plutôt semblé constituer une étape du développement du savoir-parler en public propre à nos apprenants de niveau A2.

14 Les outils du Web 2.0 sont très présents, selon les dires des enquêtés, dans leur vie à la fois extrascolaire et scolaire, et ce, depuis l'école primaire. Ceci au point que ces outils paraissent, pour certaines des personnes interrogées, inévitables, voire envahissants. Quand on les questionne sur les outils, les termes très récurrents dans leur discours sont «facile,» «facilement,» «easy,» «comfortable,» «utile,» etc. Ceux qui n'en ont pas cette maitrise sont rares, mais le mentionnent dans les questionnaires : cela crée du stress, voire de l'appréhension ou de la frustration. Ceci crée un terrain de recherche riche et ambivalent : d'un côté, ils s'approprient relativement facilement de nouveaux outils (même si c'est souvent estimé chronophage, ce qui crée de la frustration), de l'autre ces outils font partie intégrale de leur vie et ne constituent pas une nouveauté. Cependant, dans les entretiens, aucun d'entre eux ne dit les avoir utilisés d'une manière proche de ce projet en contexte d'enseignement supérieur.

15 Nous avons choisi les commentaires sur Google+ pour leur forte teneur affective, puisqu'il s'agissait de dire son appréciation des produits finals (vidéos) partagés sur cette même plateforme. Cet aspect affectif n'était pas présent dans les commentaires sur Etherpad, plus fonctionnels car visant le remue-méninges et la correction par les pairs. Pour rappel, la consigne donnée aux étudiants quant à son usage était de partager leurs productions finales, de visionner les vidéos des autres étudiants, de cliquer sur «+» pour celles qu'ils aimaient particulièrement, et de réagir en français aux trois vidéos qu'ils avaient préférées en utilisant la fonction " commentaire. " À cela s'ajoutaient, au niveau B2 seulement, de 1 à 3 points bonis pour les vidéos recueillant le plus de votes.

16 Pour la deuxième partie de nos résultats (le lien entre la réflexion/interaction affective, le Web 2.0 et la dimension significative), nous nous sommes penchées sur la quatrième question du questionnaire (voir la note 11), qui traite de l'aspect affectif ainsi qu'à la première question du questionnaire, concernant les outils, afin de voir quels étaient les rapports des apprenants avec les outils, d'une part, et si le partage et les affects y étaient abordés. Cette question était la suivante : What did you think of the use of the Web 2.0 tools (e.g. Etherpad to write and Google + to share and comment) in the activity «Mon Montréal à moi»? You can choose 2 answers. It was motivating / It was... Please explain your answer. Le questionnaire complet est en annexe.

17 Cette nuance a une certaine logique : au niveau A2, les étudiants ont encore du mal à communiquer et, du fait, peuvent se sentir inhibés. II s'agit pour eux de s'ouvrir à l'autre afin de s'en rapprocher. Au niveau B2, il y a certainement moins d'inhibition quant à la communication et avoir des sujets de conversation communs, vivre une expérience originale, aide à la création de liens.

18 L'auteur met en valeur l'importance des outils en termes de cultures d'usages : «Internet communication tools are not neutral media» (38). Comme tout artéfact humain, ils sont culturels («the cultural embeddedness of Internet communication tools» [38]) et porteurs des associations interactionnelles et relationnelles, des usages, des attentes de genre et de registre de communication.

19 Car, peut-on supposer, les apprenants sont plus prêts au niveau B2 à adopter une plus grande variété de genres discursifs nécessaires à la communication. 
Anthony, Sarah et Prisca Fenoglio. «Encourager les réflexions/interactions affectives par l'usage scénarisé des outils du Web 2.0 : en quoi est-ce significatif? " Nouvelle Revue Synergies Canada, No¹1 (2018)

\section{Bibliographie}

Arnold, Jane. «Attention to Affect in Language Learning.» Anglistik. International Journal of English Studies, vol. 22 , no. 1, 2011, pp. 11-22.

---. «Comment les facteurs affectifs influencent-ils l'apprentissage d'une langue étrangère?» ÉLA. Études de linguistique appliquée, vol. 144, no. 4, 2006, pp. 407-425.

---. Dir. Affect in Language Learning. Cambridge University Press, 1999.

Clanet, Joël. «Étude des organisateurs des pratiques enseignantes à l'université.» Revue des sciences de l'éducation, vol. 27, no. 2, 2001, pp. 327-352.

Conseil de l'Europe. Cadre européen commun de référence pour les langues : apprendre, enseigner, évaluer. Éditions Didier, 2001.

Dejean-Thircuir, Charlotte et Elke Nissen. «Évolutions technologiques, évolutions didactiques.» Le Français dans le monde. Recherches et applications, no. 54, 2013, pp. 28-40.

Dejean-Thircuir, Charlotte et François Mangenot. «Tâches et scénarios de communication dans les classes virtuelles.» Les Cahiers de l'Asdifle. Les usages des TICE en FLE/FLS, no. 17, 2006, pp. 310-321.

Dörnyei, Zoltán. Teaching and Researching Motivation. Longman, 2001.

Jézégou, Annie. «Créer de la présence à distance en e-learning. Cadre théorique, définition, et dimensions clés.» Distances et savoirs, vol. 8, no. 2, 2010, pp. 257-274.

Kreijns, Karel, et al. «Identifying the Pitfalls for Social Interaction in Computer-Supported Collaborative Environments : A Review of the Research.» Computers in Human Behaviors, vol. 19, no. 3, 2003, pp. 335-353.

Mangenot, François. «La question du scénario de communication dans les interactions pédagogiques en ligne.» Actes du colloque Jocair (Journées Communication et Apprentissage Instrumentés en Réseau). Mohamed Sidir, Éric Bruillard, G.-L Baron (dir.), 2008, pp.13-26.

Mclntyre, Peter D., et al. «Conceptualizing Willingness to Communicate in a L2 : A Situational Model of L2 Confidence and Affiliation.» The Modern Language Journal, vol. 82, no. 4, 1998, pp. 545-562.

Muller, Catherine. «La créativité dans des commentaires de photographie en classe de français langue étrangère.» Synergies Europe, no. 4, 2009, pp. 89-104.

Ollivier, Christian et Laurent Puren. Le Web 2.0 en classe de langue. Une réflexion théorique et des activités pratiques pour faire le point. Éditions maison des langues, 2011.

Piccardo, Enrica. «Évolution épistémologique de la didactique des langues : la face cachée des émotions.» Lidil, no. 48, 2013, pp. 17-36.

Puozzo Capron, Isabelle et Enrica Piccardo. « Au commencement était l'émotion : introduction. » Lidil, no. 48, 2013, pp. 5-16.

Rémon, Joséphine. «Prégnance de l'intime dans une pratique de la langue à distance : du chaos au développement langagier.» Apprendre de l'intime : entre littérature et langues. Christine CollièreWhiteside, Anne-Marie Voise, Marie Berchoud (dir.), L'Harmattan, 2016, pp. 165-186.

Rodríguez, José I., et al. «Clarifying the Relationship Between Teacher Nonverbal Immediacy and Student Cognitive Learning : Affective Learning as the Central Causal Mediator.» Communication Education, vol. 45 , no. 4 , 1996 , pp. 293-305. 
Anthony, Sarah et Prisca Fenoglio. «Encourager les réflexions/interactions affectives par l'usage scénarisé des outils du Web 2.0 : en quoi est-ce significatif ? " Nouvelle Revue Synergies Canada, No¹1 (2018)

Thorne, Steven L. « Artifacts and Cultures-of-Use in Intercutural Communication. » Language Learning \& Technology, vol. 7, no. 2, 2003, pp. 38-67.

Van Der Maren, Jean-Marie. «Les recherches qualitatives : des critères variés de qualité en fonction des types de recherche.» L'analyse qualitative en éducation. Des pratiques de recherche aux critères de qualité. Léopold Paquay, Marcel Crahay, Jean-Marie De Ketele (dir.), De Boeck Supérieur, 2010, pp. 69-84.

---. Méthodes de recherche pour l'éducation. Les Presses de l'Université de Montréal, 1995.

Zourou, Katerina. «De l'attrait des médias sociaux pour l'apprentissage des langues - Regard sur l'état de l'art.» Alsic (Apprentissage des Langues et Systèmes d'Information et de Communication), vol. 15, no. 1, 30 mars 2012, alsic.revues.org/2485. Accès le 26 juin 2017.

\section{Annexe : questionnaire / Mon Montréal à moi / hiver 2016}

1a. Do you use Web 2.0 tools (such as Instagram, Facebook, Linkedin, Pinterest, etc.) regularly?

$\square$ yes $\square$ no

1b. If so, which ones do you use and for which aspect of your life (for your studies, for communicating with friends, etc.)?

1c. What did you think of the use of the Web 2.0 tools (e.g. Etherpad to write and Google + to share and comment) in the activity "Mon Montréal à moi" ? You can choose 2 answers. $\square$ it was motivating $\square$ it was : Please explain your answer :

1d. Did this activity allow you to : - feel more comfortable with the Web 2.0 tools : $\square$ yes $\square$ no

- learn more about those tools : $\square$ yes $\square$ no

Please explain your answer :

2. How did you feel about the part of the activity that was collaborative (i.e. the script, the video)? You can choose 2 answers. $\square$ it was motivating $\square$ it was :

Please explain your answer :

3. How did you feel about the part of the activity that was creative (i.e. the script, the video, the editing)? You can choose 2 answers. $\square$ it was motivating $\square$ it was :

Please explain your answer :

4. How did you feel about the part of the activity that was affective (i.e. the personal aspect of the activity and the sharing of your feelings for a place that is special to you in Montreal)? You can choose 2 answers.

$\square$ it was motivating $\square$ it was :

Please explain your answer:

5. How did you feel about the part of the activity that was outside of the classroom (i.e. going to film a place you like in Montreal)? You can choose 2 answers. $\square$ it was motivating $\square$ it was :

Please explain your answer:

6. Were you able, through this activity, to deepen your knowledge of : $\square$ oral expression $\square$ written expression $\square$ other :

7. Finally, did you find this activity (check all that apply) : $\square$ interesting $\square$ original $\square$ useful $\square$ difficult $\square$ useless $\square$ indifferent $\square$ other :

Please explain your answer :

8. How motivated where you during this activity? 0 (not motivated) 1234 (very motivated)

Please explain your answer :

Other comment(s)? : 